binary and continuous outcomes, respectively. We performed NMA to evaluate comparative effectiveness among different complex interventions. Primary outcome was 30-day hospital readmissions, while secondary outcomes were length of stay, 30-day mortality and 30-day emergency department (ED) visits.

Results A total of six SRs and18 RCTs ( $n=3,071)$ assessing ten different complex interventions were included (IDDF2021-ABS-0182 Figure 1. Flowchart). Results of pairwise meta-analyses showed no significant difference between complex interventions and controls in reducing 30-day hospital readmissions (pooled RR: 0.93; 95\% CI: 0.73, 1.18; 16 RCTs; moderate quality of evidence), 30-day mortality (pooled RR: 1.30; 95\% CI: 0.59, 2.82; 9 RCTs; low quality of evidence) and 30-day ED visits (pooled RR: 0.81; 95\% CI: $0.55,1.21$; 2 RCTs; moderate quality of evidence). However, pairwise meta-analysis results showed that complex interventions significantly reduced length of stay (pooled MD: -2.12 days; 95\% CI: $-2.79,-1.44$ days; 14 RCTs; very low quality of evidence). NMA results indicated that there was no significant difference across different complex interventions on reducing 30-day hospital readmissions (IDDF2021-ABS-0182 Figure 2. Network plot). Nonetheless, fast-track rehabilitation might be the most effective intervention for reducing the length of stay, as supported by low certainty of evidence.

Conclusions NMA showed that complex interventions may not differ from usual care in reducing 30-day hospital readmissions among colorectal surgery patients. However, fast-track rehabilitation may have the highest potential for reducing the length of stay. The benefits of fast-track rehabilitation for colorectal surgery patients may be different across different health systems. Policymakers may consider contextual factors carefully before implementation.

\section{IDDF2021-ABS-0188 WORLDWIDE INCIDENCE AND LIFESTYLE RISK FACTORS OF GASTRIC CANCER AMONG YOUNG ADULTS: A GLOBAL STUDY}

${ }^{1} J u n j i e ~ H u a n g *,{ }^{1}$ Alfonse Ngai, ${ }^{2}$ Veeleah Lok, ${ }^{3}$ Xianjing Liu, ${ }^{4}$ Lin Zhang, ${ }^{5}$ Jinqiu Yuan, ${ }^{6}$ Wanghong Xu, ${ }^{7}$ Zhi-Jie Zheng, 'Martin CS Wong. 'Jockey Club School of Public Health and Primary Care, Faculty of Medicine, Chinese University of Hong Kong, Hong Kong; ${ }^{2}$ Department of Global Public Health, Karolinska Institute, Karolinska University Hospital, Stockholm, Sweden; ${ }^{3}$ Department of Radiology and Medical Informatics, Erasmus University Medical Centre, Rotterdam, Netherlands; ${ }^{4}$ Melbourne School of Population and Global Health, The University of Melbourne, Victoria, Australia; ${ }^{5}$ Clinical Research Centre, The Seventh Affiliated Hospital, Sun Yat-sen University, Guangdong, China; ${ }^{6}$ School of Public Health, Fudan University, Shanghai, China; ${ }^{7}$ Department of Global Health, School of Public Health, Peking University, Beijing, China

\subsection{6/gutjpl-2021-IDDF.171}

Background The purpose of this study is to investigate the incidence and lifestyle risk factors for gastric cancer in younger individuals aged 15-49 years old using global health and national cancer registry data.

Methods We retrieved the Global Cancer Observatory (GLOBOCAN) 2020 for age-standardised incidence (ASI) per 100 000 of gastric cancer in young individuals across 185 countries. We estimated the prevalence of lifestyle risk factors (smoking, alcohol drinking, and dietary habits) among the younger adults by joinpoint regression. We conducted the multivariable linear regression to examine the associations between lifestyle risk factors and incidence whilst adjusting for gross domestic products (GDP) per capita for each country.

Results The total number of new cases of young-onset gastric cancer was 100687 in 2020, with an ASI of 2.5 (IDDF2021ABS-0188 Figure 1. Worldwide incidence of young-onset gastric cancer in 2020). The highest ASI was in males (2.9 vs. 2.1 in females); Eastern Asia (3.7), Central America (2.4),

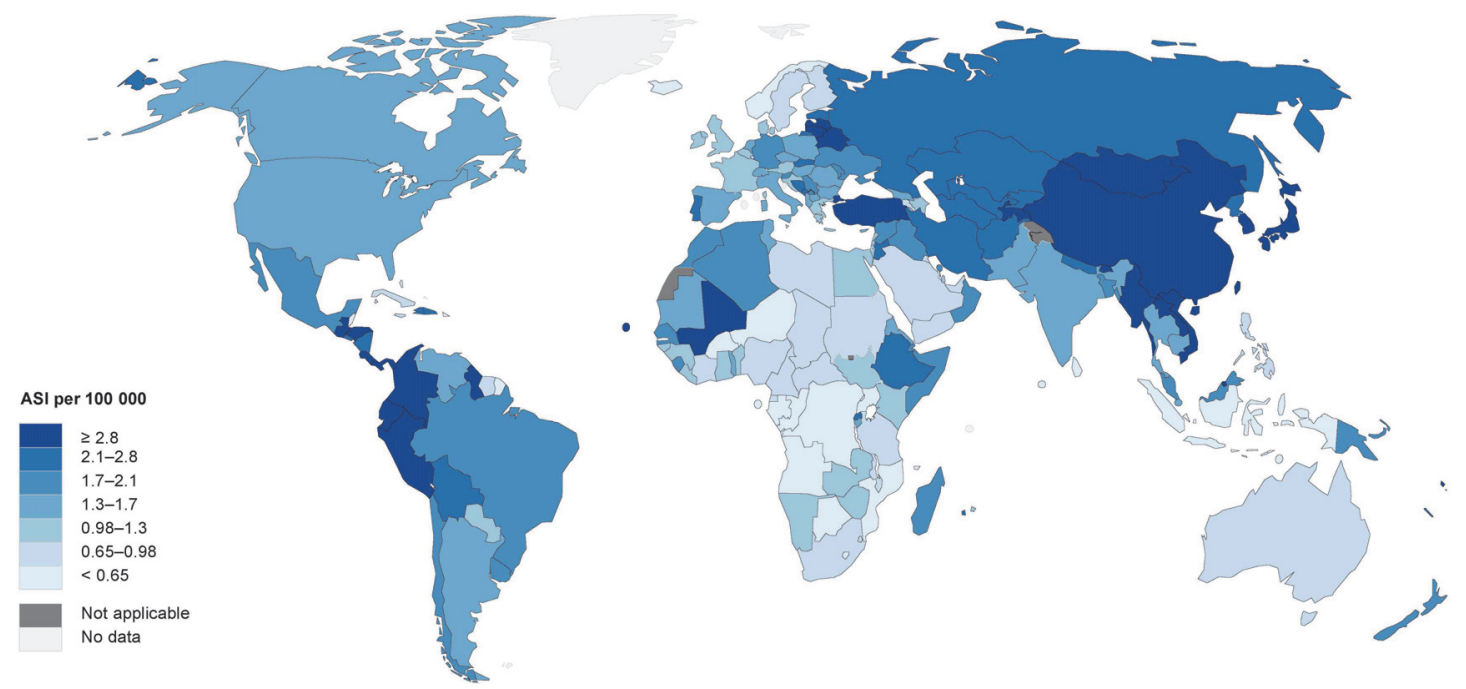

Abstract IDDF2021-ABS-0188 Figure 1 Worldwide incidence of young-onset gastric cancer in 2020. ASI, age-standardised incidence; Data source: GLOBOCAN 2020 Graph production: IARC (http://gco.iarc.fr/today) World Health Organization 
South America (2.3); and countries of Upper-Middle Income (2.8). Regions with higher incidence of young-onset gastric cancer had higher prevalence of alcohol drinking (beta coefficients $(\beta)=0.066,95 \%$ confidence intervals (CI) 0.023 to $0.109, p=0.003)$ and unhealthy dietary habits $(\beta=0.031,95 \%$ CI 0.012 to $0.050, \mathrm{p}=0.001)$, but not smoking $(\beta=0.018$, $95 \%$ CI -0.010 to $0.046, p=0.210$ ) among the young population.

Conclusions The burden of young-onset gastric cancer was substantial in 2020, with higher incidence found in male subjects, Eastern Asia, Central and South America. The associated lifestyle risk factors for young-onset gastric cancer included alcohol drinking and unhealthy dietary habits. To prevent young-onset gastric cancer, lifestyle modifications and early detection by screening for high-risk young individuals could be imperative interventions.

A OS of Patients with Left Colon Cancer

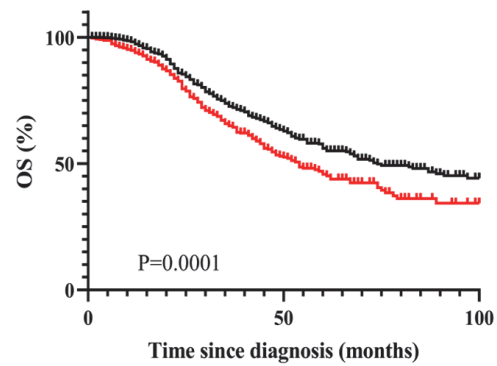

- Male (n=1102)

- Female (n=580)

A

OS of Patients wih Simultaneous Liver Metastases (All Patients, $n=1573$ )

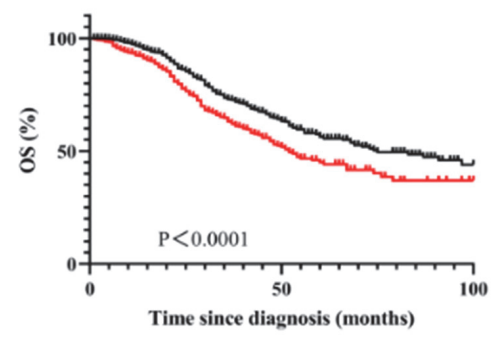

- Male (n=1042)

- Female $(\mathrm{n}=531)$

(a)

Abstract IDDF2021-ABS-0191 Figure 1

Abstract IDDF2021-ABS-0191 Figure 2

A

OS of CRCLM Patients with KRAS ${ }^{+}$ Status (All Patient, $n=461$ )
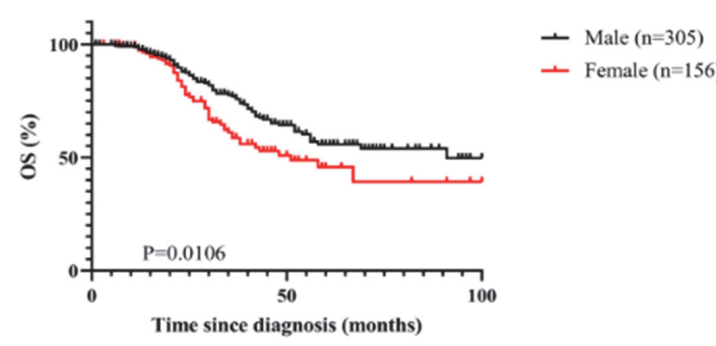

- Female (n=156)
IDDF2021-ABS-0191

GENDER MATTERS: SEX DISPARITIES IN COLORECTAL CANCER LIVER METASTASIS SURVIVAL: A POPULATION-BASED STUDY

Yibin Wu*, Weiqi Xu, Lu Wang. Fudan University Shanghai Cancer Center, China

\subsection{6/gutjnl-2021-IDDF.172}

Background Colorectal cancer with liver metastasis showed a poorer prognosis in colon cancer. The aim of this study was to investigate the impact of sex disparities in survival. The patients were stratified according to sex, age, primary tumor site, KRAS mutant status and metastatic characteristics.

Methods Patients diagnosed with colorectal cancer liver metastasis (CRCLM) between January 2007 and June 2018 at our hospital were identified. Clinical information, tumor character-

B OS of Patients with Right Colon Cancer Metastasis (All Patients, $n=550$ )

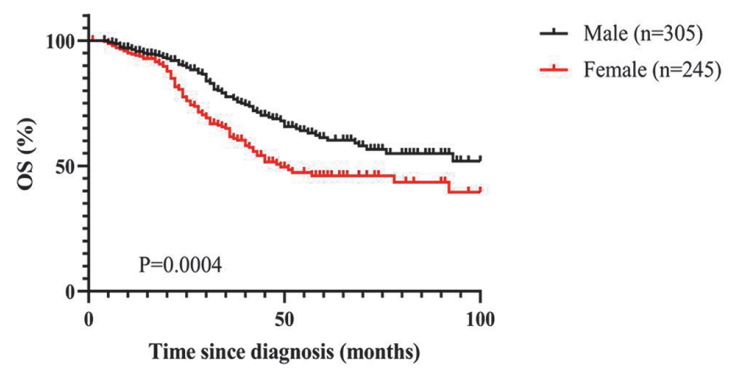

Time since diagnosis (months)

B

OS of Patients wih Metachronous Liver Metastasis (All Patients, $n=1030$ )

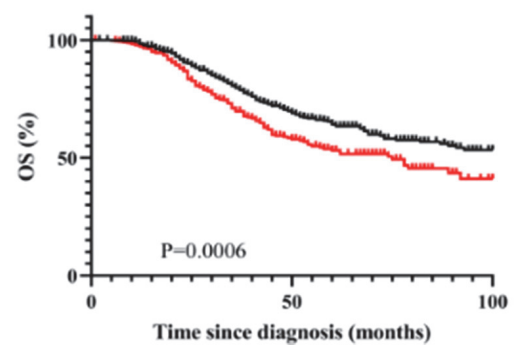

- Male (n=611)

- Female (n-419)

B

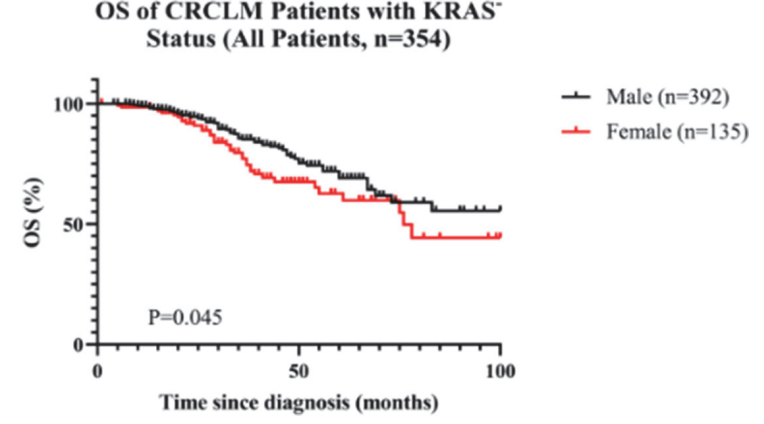

Time since diagnosis (months)

Abstract IDDF2021-ABS-0191 Figure 3 